\title{
A New Genetic Algorithm based Method for Topological Optimization of Interconnection Networks
}

\author{
P. K. Tripathy \\ Asst. Prof., Dept. of CSE, \\ Silicon Institute of Technology, \\ Silicon Hills, Bhubaneswar \\ Odisha, India
}

\author{
R. K. Dash, PhD. \\ Reader, Dept. of CSA, \\ College of Engineering \& \\ Technology, \\ Bhubaneswar, Odisha, India
}

\author{
C. R. Tripathy, PhD. \\ Professor and Head, \\ Dept. of CSE, \\ VSS University of \\ Technology, Burla, \\ Odisha, India
}

\begin{abstract}
This paper addresses the topological optimization problem of interconnection networks where the overall cost of the network is minimized subjected to a predefined network reliability constraint. A new method based on genetic algorithm is proposed which uses more efficient encoding, cross over and mutation rate to ensure a better rate of convergence towards optimal solution. The proposed method is well equipped with some new algorithms for each of the operations stated above with suitable step wise illustration. The minimum cost of fully connected networks with up to 15 numbers of nodes and 105 numbers of links are computed with respect to different targeted values of reliability. Further the optimal layout of the interconnection networks within the above computed cost are presented that meets the network reliability constraints.
\end{abstract}

\section{General Terms}

Genetic Algorithm, Optimization , Interconnection Networks,

\section{Keywords}

Interconnection Network, Genetic Algorithm, Network Reliability, Topological Optimization.

\section{INTRODUCTION}

We The design of very economical interconnection network is a complex multi constraint and multi optimization problem. Because it requires the simultaneous optimization of many objectives, they may be conflicting i.e. minimizing the cost of the network while meeting some criteria such as reliability, average delay and throughput. Out of these performance criteria the reliability may be treated as the most important performance criterion as it ensures the working of such networks in the presence of different types of failures [1]. Thus the design decision is to connect all the nodes with some known probability i.e. the network reliability while minimizing the overall cost of the network [2]. The type of topological optimization problem considering the network reliability along with other objectives such as cost is an NPhard problem [3]. This is due to exponential growth of the solution space with respect to the size of the network.

Review of literature revels mainly two approaches viz. enumerative based approach and heuristic based approach for solving this type of topological optimization problems. Some of the enumerative approaches are branch and bound [4], spanning tree [5]. The method using branch and bound technique divides the problem into several sub problems for an efficient search of the exact optimal solution. But this method is only able to find solution of the fully connected networks of maximum size of 12 nodes (60 links). Similarly, the methods based on enumeration of the spanning trees can be applied only for the small networks, as for large sized networks the computational complexity in enumerating all the spanning trees prohibits using of such techniques. So for the networks of larger size, the heuristic methods like simulated annealing [6][7][8][9], Artificial Neural Network [10], Genetic Algorithm [13][14][15][16][17] are preferred because of their strength and ability to find near optimal solutions. Pierre and et al. [7] used simulated annealing to find optimal designs for packet switched networks where delay and capacity were considered, but reliability was not. Another heuristic approach using an optimize neural network for solving the network topological optimization problem is found in [10]. Similar kind of works can be found in [11] and [12]. However mapping of the topological optimization problem onto a neural network is quite cumbersome. Further literature also records that the heuristic methods based on Artificial Neural Network may not always lead to optimal solutions. The review of literature reveals the use of genetic algorithm effectively in the past to solve many difficult engineering problems as well as the optimization problems [15][16]. Marseguerra et.al. [17] determined optimal surveillance test intervals using multi-objective Genetic Algorithm with the goal of improving reliability and availability. Elegbede and Adjallah [18] presented a multi objective combinatorial optimization method modeled with continuous and discrete variables to optimize the availability and the cost of repairable parallel series systems. Deb et al. [19] formulated a bi-objective optimization problem of minimizing total wire length and minimizing the failure rate in the printed circuit board design. They have implemented NSGA-II to solve the problem. Kumar et al. [20] presented a multi objective genetic algorithm to design telecommunication networks while simultaneously minimizing network performance and design cost under reliability constraint. The methods discussed so far though solved many optimization problems, they as such can not be able to solve the topological optimization problem of interconnection networks, where the main objective is to minimize the overall cost of the interconnection network while satisfying some reliability constraint. This motivates our study to propose a new method that uses genetic algorithm with simple and efficient chromosome encoding as well as better cross over and mutation techniques in solving such topological optimization problems in Section 2 of this paper. The rest of the paper is organized as follows: Section-3 contains the application of the proposed method on some fully connected Interconnection Networks and Section-4 concludes the paper with future scope. 
The following Notations and assumptions are used throughout this paper.

\section{Notations}

ICN Completely connected interconnection network.

$G(N, L)$

Probabilistic Graph $G$ of the given Interconnection Network having $\mathrm{N}$ number of nodes and $\mathrm{L}$ number of links.

$P \quad$ Population

$P_{s} \quad$ Population size

$C_{p} \quad$ Crossover probability

$Z \quad$ Objective function

$R(X) \quad$ Reliability of network $\mathrm{X}$

$x_{i j} \quad$ A decision variable.(1 if a link exist between node $i$ and node $j, 0$ otherwise)

$R_{0}$

Minimum reliability constraint.

$C_{i j}$

$R_{i j}$

$f(X) \quad$ Fitness of the network $x$

Max_Cost $(P) \quad$ Maximum network cost of a population $\mathrm{P}$

$\operatorname{Cost}(X)$

Cost of every network $\mathrm{x}$

P1 and P2 Selected two networks for chromosome level cross over

C1 and C2 Generated Child networks after chromosome level cross over.

Pt1; Pt2 Two nodes chosen from network

Cost $_{\text {max }}$

Maximum permissible Cost/Budget Constraint

$K \quad$ The set of nodes having degree less than 2 .

$|K| \quad$ The number of nodes in $K_{\text {set. }}$

NR Network Reliability

\section{Assumptions}

1. Nodes are perfectly reliable.

2. Links are either operational or failed.

3. Failures of links are ${ }^{S}$-independent.

\subsection{Problem Statement}

An interconnection network can be modeled as a graph $\mathrm{G}(\mathrm{N}$, $\mathrm{L}$ ), where $\mathrm{N}$ is the set of nodes (Stations, terminals or computer sites) and $\mathrm{L}$ is the communication links among them. As in practically, the nodes are far away from each other. The distance between them may vary. If the nodes of the network are fixed and the associated links are predefined, the main objective is to find out best layout of links within a minimum cost while satisfying a given reliability constraint, or mathematically the objective function is expressed as:

Minimize

$$
Z=\sum_{i=1}^{N-1} \sum_{j=i+1}^{N} C_{i j} x_{i j}
$$

Subject to

$$
R(X) \geq R_{0}
$$

And degree of each node of $G \geq 2$

\subsection{Fitness Function:}

A network will be included in the population if it satisfies two conditions:

1. Each node of the network must have at least degree 2.

2. The computed reliability of the network must be greater than the given value of the reliability constraint.

For the networks that satisfy the above conditions, the fitness function can be calculated as:

$$
F(X)=\text { Max_Cost }(P)-\operatorname{Cost}(X)
$$

\section{PROPOSED METHOD}

\subsection{Definitions}

Definition 1. Spanning Tree is a maximal set of edges of $\mathrm{G}$ that contains no cycle, or as a minimal set of edges that connect all vertices.

Definition 2. A steady state is defined as the state of a system that does not change over time or in which any one change is continually balanced by another.

\subsection{Encoding}

The proposed method uses the following variable length integer encoding string representation:

For a network with $\mathrm{N}$ number of nodes and $\mathrm{L}$ number of links, the length of the chromosomes is the maximum number of links of the network, which requires much fewer number of integers to represent the chromosome than other encoding techniques [23]. Every possible link is numbered sequentially starting from 1. 


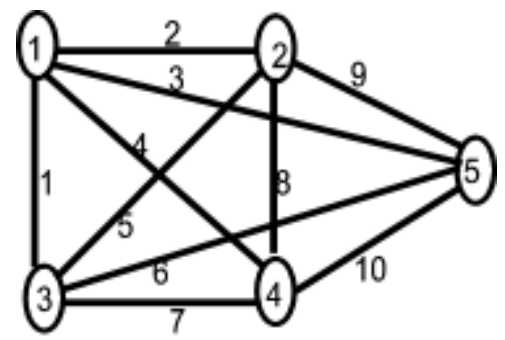

(a)

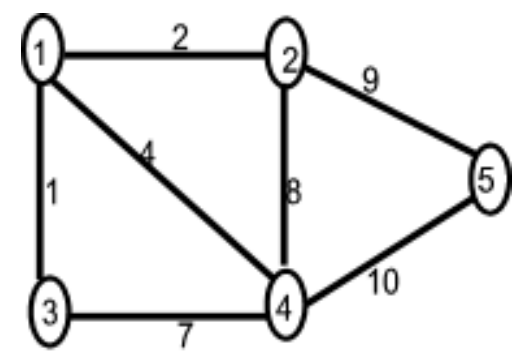

(b)

Figure 1: (a) A fully connected network with 10 links that are labeled from 1 to 10 . (b) A partially connected network with 7 links using the same labeling as (a)

Example :

Chromosome Encoding of the networks in Figure 1 are:

(a) $\{1,2,3,4,5,6,7,8,9,10\}$ and

(b) $\{1,2,4,7,8,9,10\}$

\subsection{Initial Population}

The proposed method only considers the candidate networks in the initial population that must meet the reliability constraint while minimizing the overall cost and satisfying the 2-connectivity test. Thus by enhancing the efficiency of the search for the optimal solutions.

\section{Initial Population}

Input: ICN

while (Population size is not reached) do

a) Randomly generate two spanning trees [22] from the given fully connected network

b) Make it a two connected network using repair algorithm

c) Apply crossover to generate children

d) Estimate fitness of both parents and children

e) Networks that satisfy the fitness criteria will included in initial population

End while

\subsection{Chromosome level Cross Over}

Crossover is the genetic operator that mixes two chromosomes together to form new offspring. The intuition behind crossover is exploration of new solution by exploitation of existing solutions. Chromosomes with high fitness got opportunity to be selected to take part in the crossover. The steps involved in crossover operator are present in the following algorithm:

\section{Cross Over}

Input: $\quad$ Networks $P 1$ and $P 2$

Step1 : Randomly select a common link $(i, j)$ from $P 1$ and $P 2$ and select two points $P t 1=i$ and $P t 2=j$ for cross over.

Step2: The links adjacent to Pt1 and Pt2 in P2 are assigned to one child $\mathrm{Cl}$ and the links adjacent to $P t 1$ and Pt2 in P1 are assigned to child C2

Step3 : $\quad$ Determine added links

$$
P^{\prime} 1=P 1-C 2 \text { and } P^{\prime} 2=P 2-C 1
$$

Step4: Assign $C 1=C 1 U P^{\prime} 1$ and $C 2=C 2 U P^{\prime} 2$

\section{Example :}

Let two networks P1 and P2 are selected after using some selection procedure for crossover. The two networks are shown in Figure 2.

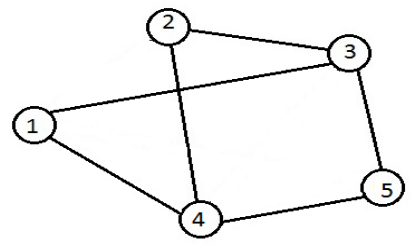

(a)

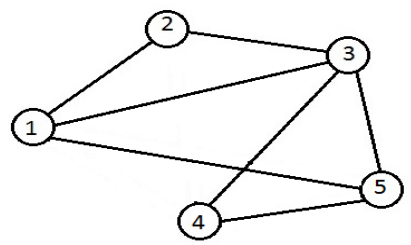

(b)
Figure 2: Two selected parent (P1 and P2) networks for crossover

Step:1 Let it chooses a common link $(2,3)$ so the two cross over points are $\mathrm{Pt} 1=2$ and $\mathrm{Pt} 2=3$.

Step:2 The links adjacent to Pt1 and Pt2 in P1 are

$\mathrm{P} 1(\mathrm{Pt} 1)=\{(2,1),(2,3)\}$ and $\mathrm{P} 1(\mathrm{Pt} 2)=\{(2,3),(1,3)$, $(3,4),(3,5)\}$

Similarly the links adjacent to $\mathrm{Pt} 1$ and $\mathrm{Pt} 2$ in $\mathrm{P} 2$ are $\mathrm{P} 2(\mathrm{Pt} 1)=\{(2,4),(2,3)\}$ and $\mathrm{P} 2(\mathrm{Pt} 2)=\{(2,3),(1,3)$, $(3,5)\}$.

Step:3 The Child $\mathrm{C} 1$ will be assigned with the adjacent links to the points of $\mathrm{P} 2$.

The links adjacent to the two points Pt1 and Pt2 in network $\mathrm{P} 2$ will be assigned to $\mathrm{C} 1$.

$\mathrm{C} 1=\{(2,3),(2,4),(1,3),(3,5)\}$

Similarly the links adjacent to the two points Pt1 and $\mathrm{Pt} 2$ in $\mathrm{P} 1$ will be assigned to $\mathrm{C} 2$.

$\mathrm{C} 2=\{(2,1),(2,3),(1,3),(3,4),(3,5)\}$ 
The children after exchanging the links adjacent to the chosen point between $\mathrm{P} 1$ and $\mathrm{P} 2$ are shown in Figure 3.

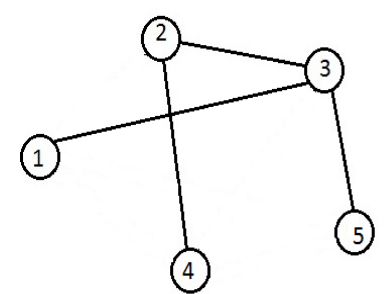

(a)

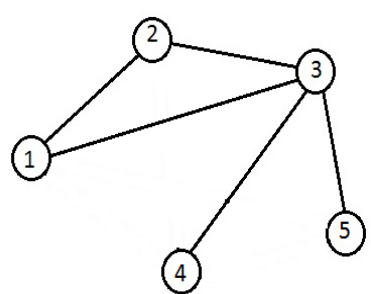

(b)
Fig 3. The intermediate generated after applying the second step of Cross Over Algorithm on P1 and P2.

Step:4 The links that are not adjacent to the Pt1 and Pt2 in $\mathrm{P} 1$ will be added to $\mathrm{C} 1$.

Added links to C1are $\{(1,4),(4,5)\}$

The links that are not adjacent to the Pt1 and Pt2 in $\mathrm{P} 2$ will be added to $\mathrm{C} 2$.

Added links to $\mathrm{C} 2$ are $\{(1,5),(4,5)\}$

The new generated off springs are shown in Figure 4.

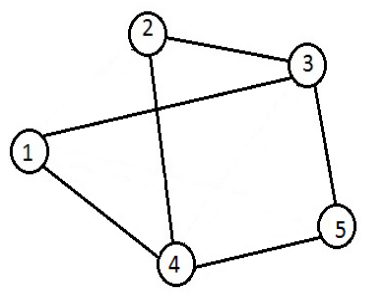

(a)

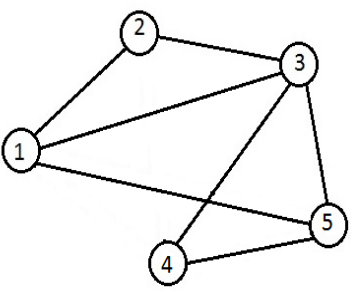

(b)
Fig 4. Two children networks $\mathrm{C} 1$ and $\mathrm{C} 2$ after applying the step 3 and step 4 of Cross Over Algorithm on P1 and P2.

\subsection{Repair Algorithm}

A repair algorithm is used here as mutation operator to add some additional links to the generated networks so as to satisfy the minimum 2-connectivity criteria. The updating is applied in the following steps:

\section{Repair Algorithm}

Input: $\mathrm{G}(\mathrm{N}, \mathrm{L})$

1. If $|K|_{=1}$ then join that node with a node having least cost.

2. If $|K|=2$ say N1 and N2 then

2.1 Compute $\mathrm{C} 1=$ The minimum cost between $\mathrm{N} 1$ and any other node $\mathrm{Nk}$ of the network.

If $\mathrm{Nk}=\mathrm{N} 2$ then join $\mathrm{N} 1$ and $\mathrm{N} 2$. else

2.2 Compute $\mathrm{C} 2=$ The minimum cost between $\mathrm{N} 2$ and any other node NL of the network.

if $\mathrm{NL}=\mathrm{N} 1$ the join $\mathrm{N} 1$ with $\mathrm{N} 2$

else

2.3 Compute $\mathrm{C}=$ Cost between $\mathrm{N} 1$ and $\mathrm{N} 2$

if $\mathrm{C}<(\mathrm{C} 1+\mathrm{C} 2)$ then join $\mathrm{N} 1$ and $\mathrm{N} 2$ otherwise join $\mathrm{N} 1$ with $\mathrm{Nk}$ and $\mathrm{N} 2$ with NL.

3. If $|K| \geq_{2 \text { then }}$

Assign a link from ICN to the existing network by joining any two nodes of $K_{\text {-set }}$ with minimum cost.

Example:

The children networks $\mathrm{C} 1$ and $\mathrm{C} 2$ satisfy the 2-connectivity criteria. But the cases may arise where the child networks may not be a 2-connected as in Figure 5.

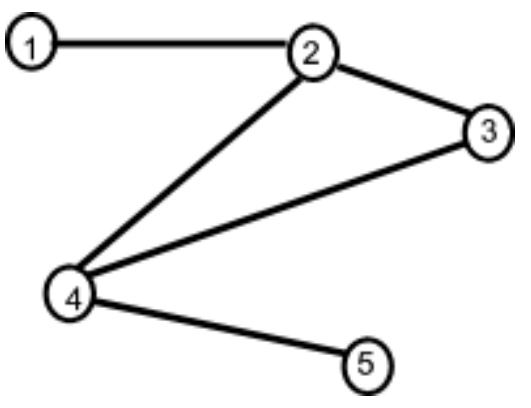

Figure 5: A network not satisfying the 2-connectivity criteria

The cost matrix of the above network is given below.

$$
\begin{aligned}
& \text { - } 25523647 \\
& \text { - } 652340 \\
& \text { - } 4329 \\
& -71
\end{aligned}
$$

The network C2 does not satisfy the 2-connectivity criteria. So the Repair Algorithm is applied on C2.

In network $\mathrm{C} 2$, node 1 and 5 has degree less than 2 , so $\mathrm{K}=\{1$, 5 \} satisfying step 2 of Repair Algorithm.

Here $\mathrm{N} 1=1$ and $\mathrm{N} 2=5$.

Step 2.1 $\mathrm{C} 1=25, \mathrm{Nk}$ is here $\mathrm{N} 2$ and $\mathrm{Nk} \neq \mathrm{N} 5$,

Step 2.2 $\mathrm{C} 5=29, \mathrm{Nk}$ is here $\mathrm{N} 3$ and $\mathrm{Nk} \neq \mathrm{N} 1$,

Step $2.3 \mathrm{C}=47$, and $\mathrm{C} 1+\mathrm{C} 2=54$

As $\mathrm{C}<\mathrm{C} 1+\mathrm{C} 2$, Node $\mathrm{N} 1$ and $\mathrm{N} 5$ will be joined.

Now the repaired network satisfying the minimum 2connectivity criteria is given in Fig. 6. 


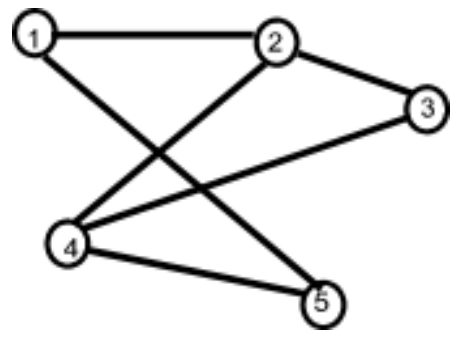

Figure 6: The example given in Figure 5 after applying Repair Algorithm

\subsection{Selection}

In selection only the fittest species can survive, breed and pass their genes to the next generation. The proposed method uses the Roulette Wheel selection algorithm for selecting individuals with a probability proportional to the fitness. The network reliability is computed using the method [21]. So the initial population taken for the example network (Figure 1) is given in Table I:

Table-I. Candidates of Initial Population

\begin{tabular}{|l|l|l|}
\hline \multicolumn{1}{|c|}{ Network } & \multicolumn{1}{c|}{ NR } & \multicolumn{1}{c|}{ Cost } \\
\hline P1 & 0.957906 & 280 \\
\hline P2 & 0.918540 & 200 \\
\hline C1 & 0.957906 & 243 \\
\hline C2 & 0.905418 & 260 \\
\hline
\end{tabular}

Applying the fitness function:

$$
F(X)=\text { Max_Cost }(P)-\operatorname{Cost}(X),
$$

The Table-II shows the selection of candidate networks for the next generation by applying its fitness function (Eq 3).

Table-II. Selection of Candidate networks based on fitness function

\begin{tabular}{|l|l|l|l|}
\hline \multicolumn{1}{|c|}{ Network } & \multicolumn{1}{|c|}{ NR } & \multicolumn{1}{|c|}{ Cost } & $F(X)$ \\
\hline P1 & 0.957906 & 280 & 0 \\
\hline P2 & 0.918540 & 200 & 80 \\
\hline C1 & 0.957906 & 243 & 37 \\
\hline C2 & 0.905418 & 260 & 20 \\
\hline
\end{tabular}

The candidate chromosomes selected for the next generation can be computed on its probability value of the fitness.

$$
\operatorname{Pr}_{i}=\frac{F_{i}}{\sum_{j=1}^{N} F_{j}}
$$

Here the networks $\mathrm{P} 2$ and $\mathrm{C} 1$ are found to be fittest for the next generation.

\subsection{Proposed algorithm}

The proposed algorithm to solve optimization problem as formulated in Section 1 is presented below:

Optimize Network

Input: (ICN, $G$, Cost_mat, Rel_mat, $\operatorname{Cost}_{\max }$ )

1. Generate initial population

2. While (steady state is not reached) do

2.1 Apply selection process with crossover probability to select chromosomes into mating pool.

2.2 Apply crossover to get new solution network

2.3 Apply repair algorithm to ensure two connected network

$2.4 \quad$ Find fitness using fitness function $f(X)$

2.5 Select the fittest chromosomes for next generation.

3. End while

4. Declare the chromosome having minimum cost.

\section{RESULT AND DISCUSSION}

For the purpose of simulation, the following parameters are set:

Population size $(\mathrm{Ps})=10$, Crossover probability $(\mathrm{Cp})=0.8$, the mutation rates from 0.002 to 0.044 and the reliability of each link $=0.9$. The costs of the links are set as per [16].

The considered mutation rate is in average smaller than its maximum value, which ensures the preservation of the original network.

The cost of few fully connected interconnection networks having up to 15 numbers of nodes are computed under different reliability constraints (Table III).

Further, the optimized networks satisfying the different reliability constraints thus generated for different input networks are presented in the figure 7 . 
Table-III. Application of proposed algorithm on some sample Networks

\begin{tabular}{|c|c|c|c|c|c|c|c|}
\hline $\begin{array}{c}\text { SI. } \\
\text { No }\end{array}$ & $\mathbf{N}$ & $R_{0}$ & $\begin{array}{c}\text { Computed } \\
\text { Optimum } \\
\text { Cost } \\
\text { Of the } \\
\text { Network }\end{array}$ & $\begin{array}{c}\text { NR of the } \\
\text { Optimum } \\
\text { Network }\end{array}$ & $\begin{array}{c}\text { CPU } \\
\text { Time in } \\
\text { sec } \\
{[16]}\end{array}$ & $\begin{array}{c}\text { CPU Time } \\
\text { in sec using } \\
\text { proposed } \\
\text { method }\end{array}$ & $\begin{array}{c}\text { Optimum } \\
\text { Network } \\
\text { obtained } \\
\text { using } \\
\text { proposed } \\
\text { method }\end{array}$ \\
\hline 1 & 5 & 0.9 & 167 & 0.9774075 & $*$ & 11.3599 & Fig. 7(a) \\
\hline 2 & 6 & 0.9 & 188 & 0.9672261 & 52.39 & 19.2113 & Fig. 7(b) \\
\hline 3 & 7 & 0.9 & 260 & 0.9556194 & 135.98 & 23.9944 & Fig. 7(c) \\
\hline 4 & 8 & 0.9 & 275 & 0.9637000 & 260.86 & 59.1566 & Fig. 7(d) \\
\hline 5 & 9 & 0.85 & 295 & 0.9287880 & 378.91 & 102.838 & Fig. 7(e) \\
\hline 7 & 11 & 0.85 & 429 & 0.8771490 & 1532.3 & 367.799 & Fig. 7(f) \\
\hline 7 & 12 & 0.85 & 459 & 0.9115021 & $*$ & 1098.12 & Fig. 7(g) \\
\hline
\end{tabular}

* Not computed in [16].<smiles>O1OC2OOC1O2</smiles>

(a)
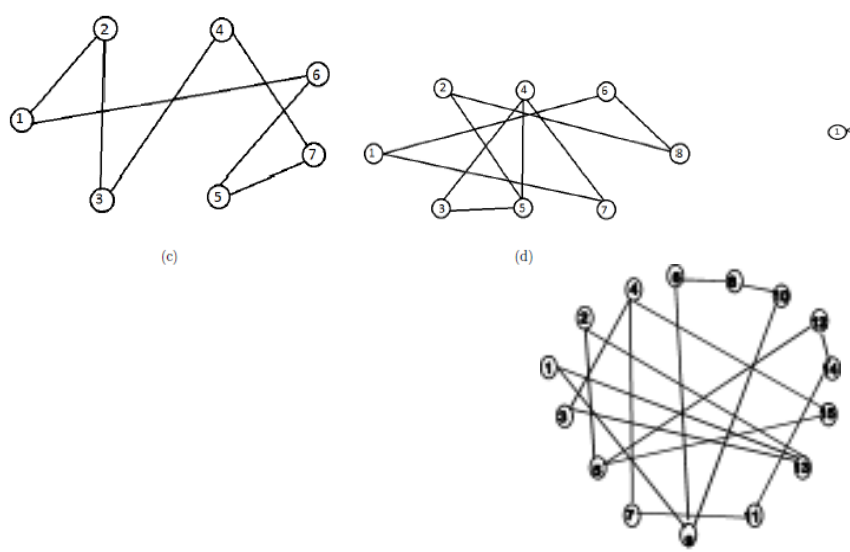

(h)
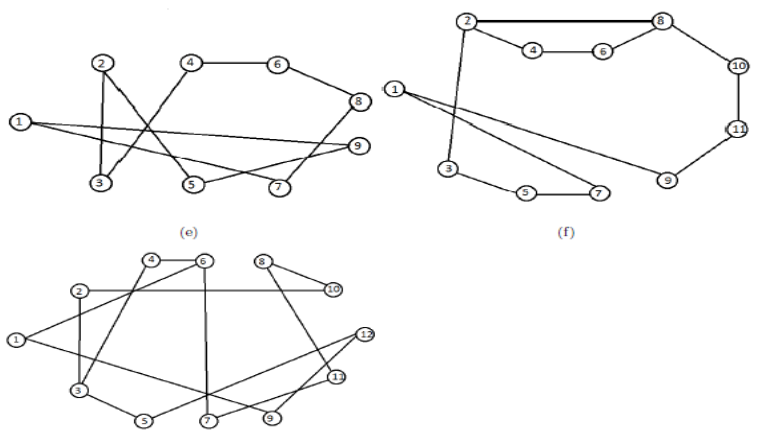

(f)

Figure 7: The optimal networks obtained after applying the proposed algorithm.

The proposed method is compared against method [16] in terms of Cpu time taken to get optimal solutions for different input networks of same interest. From this comparison it is quite clear that the proposed method requires much less $\mathrm{Cpu}$ time in minimizing the overall cost of the network under different reliability constraints.

From, Table III and Figure. 7, the following observations can be made:
For the optimized network having 7 numbers of nodes, the cost of the network is computed to be 260 within 23.99440 CPU seconds while the value of the reliability is 0.9556194 . The optimized network with 11 numbers of nodes is generated within $367 \mathrm{Cpu}$ secs whose reliability and the minimal cost is computed to be 0.8771490 and 429 respectively. However, for a comparatively larger sized network with 15 numbers of nodes and 105 numbers of links, the optimized network is 
generated in a cpu time of 1205 secs with a cost of 644 and reliability of 0.986545 .

From the different observations as discussed above, it can be ensured that the proposed method generates an optimized network with a minimal computed cost while meeting the reliability constraint.

\section{CONCLUSION}

This paper presents a genetic algorithm based method to address a topological optimization problem of interconnection network to minimize the overall cost of the interconnection network while satisfying some reliability constraint. The proposed method uses an efficient variable length chromosome encoding technique with chromosome level cross over to ensure a better convergence towards optimal solution i.e. generation of a network having the least cost. Further, a Repair algorithm is proposed for the situation when the generated networks are not 2-connected. The simulated results reveals the proposed method to be very efficient and less time consuming in optimizing the cost of different interconnection networks with in different reliability constraints. The works carried out in this paper can be further extended to solve similar topological optimization problems with additional complexity like network delay, throughput etc. as objective and/or constraints.

\section{ACKNOWLEDGMENTS}

Sincere thanks to Mr. Ipsit Hota, student of MTech. (CS), Silicon Institute of Technology; who helped us in Matlab implementation of the proposed work. We are also thankful to the experts who have contributed towards review of our research article.

\section{REFERENCES}

[1] Colbourn C. J. 1987 The Combinatorics of Network Reliability: Oxford Univ. Press.

[2] Coit D. W., Jin T. and Wattanapongsakorn N. 2004 System Optimization with Component Reliability Estimation Uncertainty: A multi-Criteria Approach, IEEE Transaction On Reliability, 53(3): 369-380.

[3] Garey M. R. and Johnson D. S. 1979 Computers and Intractability: A Guide to the Theory of NPCompleteness: W. H. Freeman \& Co.

[4] Jan R. -H., Hwang F.-J., and Cheng S.-T., 1993 Topological optimization of a communication network subject to reliability constraint. J IEEE Transaction on Reliability, 42(1): 63-70.

[5] Aggarwal K. K., Chopra Y.C., and Bajwa J.S. 1982 Topological layout of links for optimizing the overall reliability in a computer communication system. J Micro electron and Reliability, 22( 3): 347-351.

[6] Coan B. A., Leland W. E., Vecchi M. P., Wwnrib A., and Wu L. T. 1991 Using distributed topology update and preplanned configurations to achieve trunk network survivability. J IEEE Transactions on Reliability, 40(4): 404-416.

[8] Rose C. 1992 Low mean intermodal distance network topologies and simulated annealing. J IEEE Transaction on Communications, 40(8): 1319-1326.
[9] Van Laarhoven P. J. M., and Aarts E. H. L. 1987 Simulated Annealing: Theory and Applications. D. Reidel, Dordrecht, Holland.

[10] AboElFotoh Hosam M. F., and Al-Sumait Loulwa S. 2001 A neural Approach to Topological Optimization of Communication Networks with Reliability Constraints. J IEEE Transactions on Reliability,50(4): 397-408.

[11] Altiparmak F., Dengiz B. and Smith A. E. 2003 Reliability Estimation of Computer Communication Networks: ANN Models. Proc. Eighth IEEE international Symposium on Computers and Communication, 2: 1353-1358

[12] Ali M. K. M. and Kamoun F. 1993 Neural Networks for shortest path computation and routing in computer networks. J IEEE Transactions on Neural Networks, 4(6): 941-955.

[13] Coit D. W. and Smith A. E. 1996 Reliability Optimization of Series-Parallel Systems using a Genetic Algorithm. J Computers and Operation Research, 23(6): 515-526.

[14] Cox L. A., Davis L., and Qiu Y. 1991 Dynamic anticipatory routing in circuit-switched telecommunication networks. Handbook of Genetic Algorithms, New York: Van Nostrand Reinhold.

[15] Dengiz B., Altiparmak F., and Smith A. E. 1997 Local Search Genetic Algorithm for Optimal Design of Reliable Networks. J IEEE Transactions on Reliability,1(3):179-188.

[16] Dengiz B., Altiparmak F., and Smith A. E. 1997 Efficient Optimization of All-Terminal Reliable Networks, Using an Evolutionary Approach. J IEEE Transactions on Reliability,46(1):18-26.

[17] Marseguerra M., E. Zio, Podofillini L. 2004 Optimal reliability/availability of uncertain systems via multi objective genetic algorithm. J IEEE Transaction Reliability,53(3): 424-434.

[18] Elegbede C., Adjallah K. 2003 Availability allocation to repairable systems with genetic algorithms: a multi objective formulation. Reliability Engineering and System Safety, 82( 3): 319-330.

[19] Deb K., Jain P., Gupta N. K., Maji H.K. 2004 Multi objective placement of electronic components using evolutionary algorithms. J IEEE Transactions on Components Packaging Technology, 27(3): 480-492.

[20] Kumar R., Parida P. P., Gupta M. 2002 Topological design of communication networks using multi objective genetic optimization, Proceedings of World Congress on Computational intelligence, 1: 425-430.

[21] Tripathy P. K., Dash R. K. and Tripathy C. R. 2010 The reliability of the interconnection networks through self generating disjoint minimal cut-set method. IEEE 4th International Symposium on Advanced Networks and Telecommunication Systems (IEEE ANTS): 97-99.

[22] Cormen T. H., Leiserson C. E. and Rivest R. L. 1990 Introduction to Algorithms, MIT Press.

[23] Deeter Darren L., Smith Alice E. 1997 Heuristic Optimization of Network Design Considering Allterminal Reliability. Proceedings of Annual Reliability and Maintainability Symposium:194-199. 